\title{
CALIBRATION AND STANDARDIZATION ISSUES FOR THE OPTICAL TIME-DOMAIN REFLECTOMETER
}

B.L. Danielson

National Bureau of Standards U.S. Department of Commerce

Boulder, Colorado 80303-3328

December 1987

$-Q C$
100
.456
$87-3078$
1987





\section{CALIBRATION AND STANDARDIZATION ISSUES FOR THE OPTICAL TIME-DOMAIN REFLECTOMETER}

\section{B.L. Danielson}

Electromagnetic Technology Division

Center for Electronics and Electrical Engineering

National Engineering Laboratory

National Bureau of Standards

Boulder, Colorado 80303-3328

December 1987

Sponsored by

Department of the Navy

Naval Weapons Station/Seal Beach

Corona, California 91720-5000

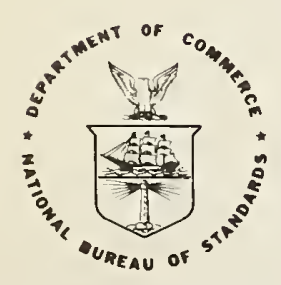

U.S. DEPARTMENT OF COMMERCE, C. William Verity, Secretary 

Contents

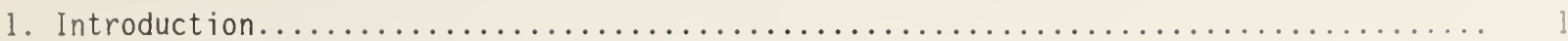

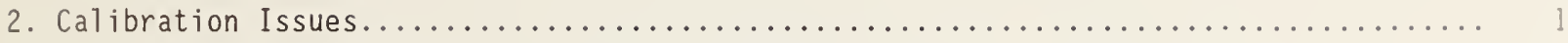

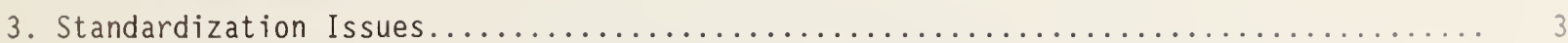

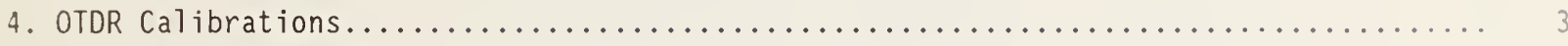

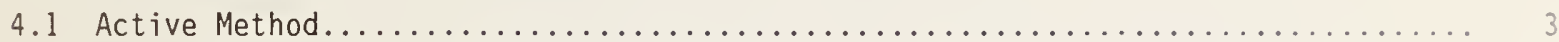

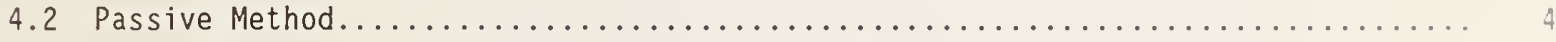

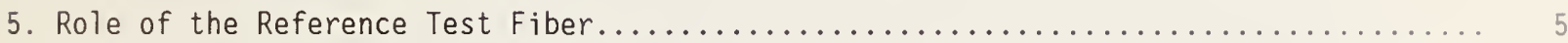

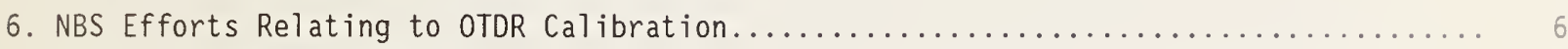

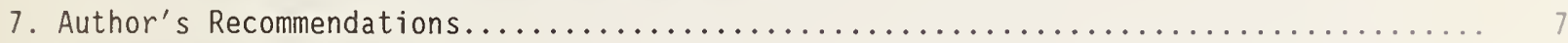

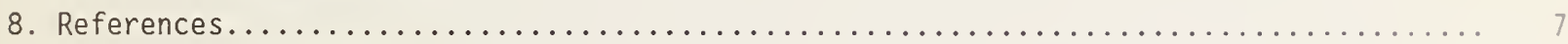



Calibration and Standardization Issues for the Optical Time-Domain Reflectometer

\section{B. L. Danielson}

National Bureau of Standards

Boulder, Colorado 80303

We review some of the issues related to the specification and assurance of optical time-domain reflectometer (OTDR) performance. These include selection of appropriate performance parameters, definition of terms, test procedures, measurement difficulties, and use of standard reference fibers. Some recommendations are given for an OTDR calibration program.

Key words: calibration procedures; optical time-domain reflectometer; OTDR; performance calibrations; standard reference fiber; standard test fiber; text procedures

\section{Introduction}

Since its inception in 1976 [1,2], the OTDR has developed rapidly into one of the most widely used and versatile test instruments for diagnostics in optical fiber communication systems. However, performance evaluation of these devices and related standards issues have lagged behind the technical development of the instrument itself. In this report we will attempt to review the current status of calibration efforts and to make some recommendations on improving and expanding these efforts in the future.

We assume some familiarity with the principals of operation of the OTDR. Readers new to the field may want to refer to review articles on the subject by Rourke [3], and Healey [4].

\section{Calibration Issues}

The calibration of an OTDR is, in general, distinct from the testing of specifications such as resolution, dynamic range, pulse overload recovery time, and signal averaging time. Specification comparisons are important when purchasing decisions must be made which involve competing instruments. Often these comparisons are possible without actually obtaining exact knowledge of the magnitude of the parameters themselves. Also, quality control or performance monitoring over a period of time may be affected without exact quantitative information. In this report we will emphasize the more precise measurements required for OTDR calibration. In our context, calibration refers to the documented measurement and uncertainty estimate of some physical quantity which is ultimately traceable to national standards. In the present case the 
only relevant quantities which fit this definition are distance and loss. An implication of this process of calibration is that measurements are made through consensus procedures arrived at by agreement with industry or military standards groups, and NBS. As mentioned, it is desirable to have the relevant measurements traceable in some way to such standards as optical power, time interval, or length.

The distance measuring accuracy or optical loss calibration of an OTDR involves a number of problems which are unique to this instrument. The most important consideration stems from the fact that the instrument does not measure the desired physical quantities directly. With an OTDR all estimates of length and loss are obtained from the analysis of features on the Rayleigh backscatter signature. Discrete loss (such as occurs at a splice, connector or defect, expressed in $\mathrm{dB}$ ), or attenuation (distributed over a fiber or cable, and expressed in $\mathrm{dB} / \mathrm{km}$ ), are quantities not measured as ratios of optical power, but inferred from observed backscatter changes in a sample under test. Also fiber length, or distance to a signature feature, is calculated from time interval measurements and must take into account the group velocity of the test waveguide. The group velocity is a function of the test fiber and wavelength.

There are many possible examples of erroneous or misleading results which can be obtained from OTDR measurements. A fiber that does not have a uniform backscatter factor will not yield a correct value for either distributed or discrete loss. Measurements made from one end of the test fiber (the usual situation) will be inaccurate if the guide does not have directional loss symmetry. Loss precision depends on the signal-to-noise ratio, so that overall uncertainties are a function of location along the fiber and probe pulse duration. Length determinations on a fiber or system of concatenated fibers with nonconstant group velocity will involve errors. These effects occur even if the instrument itself is error free i.e., is properly "calibrated". These limitations are well documented $[5,6,7]$. In some cases loss discrepancies can be minimized by taking measurements from both ends of the test fiber, but this obviates one of the principal advantages of the OTDR, namely that access to only one end of the fiber or cable is required. Methods have been proposed for reducing some types of these errors by appropriate signal analysis [8,9]. Nevertheless, since the backscatter method is based on operator interpretation of a test signature, it is plagued with instrumentindependent measurement ambiguities and uncertainties. It is well to keep these in mind in the following discussions. Also, we point out that the OTDR is not the most accurate method of measuring any physical quantity, and has not been accepted by the Electronics Industries Association (EIA) as the primary method for measuring loss. (The European standards group CCITT has recognized the backscatter method as an "alternate test method").

As a result of the difficulties mentioned above, it should be clear that calibration of an instrument does not insure accurate determinations of distance or loss (or any other quantity). The process of calibration therefore has meaning only in the sense of meeting certain performance requirements under very specific test conditions. 
In order for the various military standards laboratories to deal with instrument calibration problems in a coordinated manner, we require a solid foundation for the entire metrology program. Toward this end, we must adopt a uniform terminology and tolerances for specifications, develop consensus test procedures, and consider the desirability of accepting certain special types of reference fiber for the test measurements. Various definitions for OTDR specifications have been proposed [10-13], but as yet there is no universal agreement on these. Also, preliminary documents concerning specification test procedures [11,12] have been promulgated. As far as any reference test fiber is concerned, the EIA has decided to take no action on this matter, although they are prepared to recognize "special-use fibers" which could include the sort of devices which we feel are necessary for precise OTDR calibration. We will elaborate on these considerations in the discussion which follows.

\section{OTDR Calibrations}

A calibration procedure normally consists of performance checks, and, if possible, adjustment of the device under test to bring the instrument into compliance with predetermined specifications. The latter part of this procedure depends on manufacturer's recommendations and can not be considered here. We also require that all tests be made without internal access to the instrument. There are two basic approaches which can be used for performance checks. These we will refer to as the active and passive techniques. The former involves testing the components of the OTDR (1aser source, output coupler, and signal processing electronics), while the latter uses a reference test fiber to sample performance responses of the OTDR at a limited number of selected points.

\subsection{Active Method}

A11 OTDRs have a laser source (usually pulsed) used in conjunction with a directional coupler. The output of the coupler is connected to a common receive/transmit (R/T) port which serves to both 1 aunch power into the test fiber and return the backscattered power to the detector. The optical guide under test is connected to this port usually by means of a standard bulkhead connector. The properties of the instrument are characterized in two separate configurations.

In the first configuration, shown in Figure 1 (a), the output from the laser-coupler combination is analyzed. Properties which are recorded include operating wavelength, repetition rate, risetime, optical pulse duration (which affects resolution), and optical pulse energy (which determines, among other quantities, the dynamic range). These measurements can be made in a straightforward way with calibrated detectors for power and energy, a calibrated spectrometer or optical spectrum analyzer for wavelength, and a frequency counter for the repetition rate. Connection of OTDR and test instruments is preferably done with a standard fiber, since in general the coupled power is dependent on the type of fiber employed. 
The second configuration, one variation of which is shown in Figure $1(b)$, allows for complimentary probing of the detector-coupler-signal processing components. Here, known levels of power, simulating the backscatter from a test fiber, are injected into the $R / T$ port. Careful control of power levels can yield determinations of scale linearity (in $d B$ ) and therefore a calibrated signature response. Also, the noise floor (needed for the dynamic range) can be measured. These measurements require an optical signal generator, and calibrated attenuator. Detailed procedures for loss calibration are in some cases given by the OTDR manufacturers [14]. The time scale calibration can sometimes be accomplished by injecting optical pulse trains of known repetition frequency. Since the OTDR range scale is expressed in terms of distance, not time, the mapping of time into distance is done by the OTD using the group index which can be selected by the operator. Rickenbach and Wechsler [15] provide an example of the time and loss measurements. Calibration procedures must be tailored to the type of instrument under test. For example, methods illustrated in Figure 1 do not apply directly to coherent reflectometers, reflectometers which use correlation (pulse coding) techniques, or high-resolution instruments that use sampling oscilloscopes. Several such instruments are available commercially.

It should be noted that, in order for the OTDR data processing electronics to display range properly, the power injected into the $R / T$ port has to be synchronized with the launch of the probe pulse. This may require some user-supplied electronics. Also, externally injected pulses travel one way, while the OTDR timing circuitry is based on round-trip travel.

Of course, a11 test instruments used in the characterization of the OTDR must themselves be properly calibrated and traceable to appropriate standards.

\subsection{Passive Method}

A standard reference fiber provides a simple alternative to active calibration techniques Here, a fiber is characterized as to loss and length and is used to check the OTDR response at selected points. The fiber loss is measured by transmission measurements, which are more accurate than the corresponding reflectometry determinations. The reference fiber length is also carefully documented by independent methods. Crucial to this approach is the accurate determination of group velocity. After the fiber has been well characterized, it can be used to generate a reference test pattern on the OTDR display. Since all reflectometer measurements depend ultimately on interpretation of a backscatter signature, the known properties of the fiber can be used to estimate the errors in the OTDR analysis of these properties.

There are some drawbacks to this approach, including the following:

a) The test fiber must be uniform and must be accurately characterized. It is not always an easy task to procure and evaluate such a standard. This problem is particularly acute in the case of multimode fibers which have transient loss properties.

b) The backscatter data occur only over a relatively restricted distance, since it is not practical to make the fiber longer than a few $\mathrm{km}$. The end point provides the only length 
calibration point. The latter obstacle can be overcome by constructing a recirculating delay 1 ine out of the fiber $[10,16]$. This structure generates a comb of calibrated distance markers but the presence of a coupler in the system may produce uncertainties in the loss calibration.

c) Properties other than distance measuring accuracy and attenuation are not easily incorporated into the reference signature. Nevertheless, efforts in this direction have been at tempted.

d) In case of reflectometer malfunction or failure, this method offers no clue as to possible causes of the trouble.

Advantages inherent in this approach include:

a) No additional equipment is required for calibration of distance measuring accuracy or attenuation.

b) It is particularly convenient for routine checking of OTDR performance, for example after field use in hostile environments.

Both DESC [11] and BELLCORE [12] have adopted control fibers as part of their specifications testing programs.

Details on fiber end preparation, launch conditions, length and attenuation measurements and group index determinations (if unknown) are given in references 11-13.

5. Role of the Reference Test Fiber

A standard (or reference) test fiber (STF) can be useful in two distinct applications. The first is in the capacity of joining terminal equipment. The second application is as a well-characterized fiber which can be used as a standard reference material to help calibrate not on 1y OTDRs but other test instruments as we11.

An example of the use of a standard fiber for connecting test instruments has been given in Figure $1(\mathrm{a})$. For illustrative purposes in this case, let us assume multimode transmission paths throughout this system, and that the launch at the R/T port overfills the connecting optical cable. Also, assume that the receiver accepts all power transmitted through the jumper. In that case the amount of power registered by the optical receiver is proportional to the square of the fiber diameter and numerical aperture [10]. Obviously, the recorded power depends on the specific properties of the optical fiber selected. Although with single-mode fibers modal problems do not arise, excess loss can occur due to mode field diameter mismatches at the points of connection on either side of the jumper. Here again we are forced to conclude that a reference fiber is required if we are to obtain reproducible measurements of power and energy from such a configuration. Similar conclusions are warranted if the connection consists of non-standard fibers. Optical power can also be propagated in the cladding and/or highly attenuated modes which may be significant for certain types of coatings and transmission lengths. In this case mode strippers of some kind may be required. 
A second example illustrating the necessity for a STF is in the calculation of dynamic range. Dynamic range of an OTDR is often defined as the ratio of the backscattered power (at the launch end of the test fiber) to the noise floor. However, the peak backscattered power is a function of the transmission properties of the fiber [10]. If a physical reference fiber is used (passive method), this quantity may be determined experimentally. If the active approach is taken, dynamic range must be calculated from theoretical relations [10] using some preselected reference values for the assumed test fiber. Again, specifications are more fiber-dependent when we are dealing with multimode fibers, but variations occur even in the single-mode case.

It should be clear that, however one makes power measurements or arrives at specifications such as dynamic range, reproducible and consistent results can be achieved only with lightguides having specific, well-defined properties. In some cases these properties (e.g. NAA, core diameter, mode field diameter, index profile parameter) may simply be paper standards. In other cases it is more appropriate to have physical reference fibers.

\section{NBS Efforts Relating to OTDR Calibration}

At the current time there are several projects being conducted at NBS which impact on the accurate evaluation of OTDR performance. We will briefly touch on some of these.

First, we mention that the national standards of power and energy are maintained at the Boulder laboratories. Traceability to these standards will be necessary for calibration of some of the test instruments required on the active methods described in section 4 . At present, we are developing more sensitive standards (less than $1 \mathrm{mw}$ average power).

Some exploratory work has been conducted on passive test fixtures for evaluation of some of the performance parameters used by instrument manufacturers in the specification of commercial instruments. These test fixtures generate a reference signature from which certain OTDR responses may be inferred. In particular, distance markers may be used to calibrate the length scale of the OTDR. At their present state of development, the usefulness of these devices is restricted to comparison of competing OTDR models and monitoring performance over a period of time. Loss determinations are not sufficiently accurate to be used for calibration purposes.

Facilities are in place for measuring spectral attenuation and other properties of single mode fibers at the most common wavelength ranges. These may be used in reference test fibers.

Actual distance measurements on an OTDR require a knowledge of the group velocity of the test fiber. A program currently under way at NBS is concerned with developing an interferometric technique for measuring the group index of a small sample of the test fiber to an expected accuracy of about 0.05 percent. We are also investigating the relative accuracies of measuring fiber length by time-of-flight and mechanical means. 
We believe that startup of any effective calibration program must be preceded by proper groundwork. In that context we offer the following recommendations:

a) A first step in developing calibration facilities is the establishment of priorities in selection of performance parameters, type of fiber to be tested, wavelength of operation, acceptable tolerances, and so forth. There are quite a few possibilities here, and calibration strategies will depend on particular system requirements. At the present time distance measuring accuracy and/or distributed attenuation seem to us to be the most important properties as well as the quantities most susceptible to accurate measurements.

b) We need to establish agreement in OTDR specification terminology apart from any calibration of these devices. These terms include resolution, dynamic range, distance measuring accuracy, and overload recovery time (dead time). The consensus must be achieved through standards groups in cooperation with instrument manufacturers, military standards groups, and possibly other user's organizations.

c) Uniform procedures need to be developed for measuring the specifications and performance parameters. We feel that a reference test fiber is an important component of this process. Adoption of any procedures should also be coordinated with appropriate standards organizations.

d) The military standards calibration laboratories must decide on the best approach, active or passive, for calibrating the reflectometers. While use of a reference test fiber may be adequate as an interim measure or if distance measurement is the only consideration, we feel that military standards laboratories should plan on establishing complete active calibration facilities. This will require a calibrated (with respect to wavelength and power) optical source, time interval counter, calibrated optical spectrum analyzer, and probably a calibrated attenuator or low-level detector.

e) Military testing laboratories should base specifications and test methods on the MILSPECS [11] and FOOTPP-59 (EIA-455-59) [13] documents .

f) NBS should become involved in the last step of the calibration process, which is providing the necessary traceability to national standards.

\section{References}

[1] Barnoski, M.K.; Jensen, S.M. Fiber waveguides: A novel technique for investigating attenuation characteristics. Appl. Opt. 15(9): 2122-2115; 1976 September.

[2] Personik, S.D. Photon probe-an optical time-domain reflectometer. Bell syst. Tech. J. 56(4):355-366; 1977 March.

[3] Rourke, M.D. An overview of optical time-domain reflectometry, in Physics of Fiber Optics, vol. 2 Advances in Ceramics, B. Bendrow and S.S. Mitra eds. Columbus, OH: American Ceramic Society, Inc. 1980; 252-272.

[4] Healey, P. Review of long wavelength single-mode optical fiber reflectometer techniques J. Lightwave Technol. LT-3 (4): 876-886; 1985 August. 
[5] Matthijsse, P.; De Blok, C.M. Field measurement of splice loss applying the backscattering method. Electron. Lett. 15(24): 7795-797; 1979 November 22.

[6] Danielson, B.L. An assessment of the backscatter technique as a means for estimating loss in optical waveguides. Nat. Bur. Stand. (U.S.) Tech. Note 1018; 1980 February; $76 \mathrm{p}$.

[7] Nakahira, M.; Tokuda; M.; Omote, K.; Uchida, N.; Fukutomi, H. Measurement of optical fiber loss and splice loss by backscatter method. Trans. IECE Japan, 63(E)(10): 762-767; 1980 October.

[8] Di Vita, P.; Rossi, U. Backscatter measurements in optical fibers: separation of power decay from imperfection contribution. Electron. Lett. 15(15): 467-469; 1979 July 19.

[9] Kapron, F.; Kozikowski, C.; Crotts, R. Mode field diameter effects on OTDR splice measurements. Technical Digest-Symposium on Optical Fiber Measurements, 1986. NBS Special Publication 720 .

[10] Danielson, B.L. Optical time-domain reflectometer specifications and performance testing. App1. Opt. 24(15): 2313-2322; 1985 August.

[11] Proposed military specification, optical time domain reflectometer(OTDR), portable, militarized, for field inspection of optical Electronics Supply Center. Directorate of Engineering Standardization, Dayton, OH, 45444-5282. Draft Date 1987 February 18.

[12] Technical Advisory, Optical Time Domain Reflectometers. Technical Reference TR-TSY-000196, Issue 1, October 1986. Bell Communications Research, Inc. Morris Research and Engineering Center, 435 South St. Room 2K174, Morristown, NJ, 07960-1961.

[13] FOTP-59 (EIA-455-59) Use of the OTDR for fiber point defects. FOTP-XX (EIA-455-XX) Use of the OTDR for fiber of cable attenuation. FOTP-YY (EIA-455-YY) Use of the OTDR for fiber or cable length. Electronics Industries Association, Engineering Dept. 2001 Eye St., N.W., Washington, D.C. 20006.

[14] For example: Tektronix Operator's Manual for OF150 Optical Time-Domain Reflectometer. Section 5. May 1982.

[15] Rickenbach, R.; Wechsler, E.R. Verifying OTDR calibration quickly. Laser and Applications 4(2): 77-82; 1985 February.

[16] Nazarathy, M.; Newton, S.A. Rayleigh backscattering in optical fiber recirculating delay 1ines. App1. Opt. 25(7): 1051-1055; 1986 Apri1 1. 
(a)
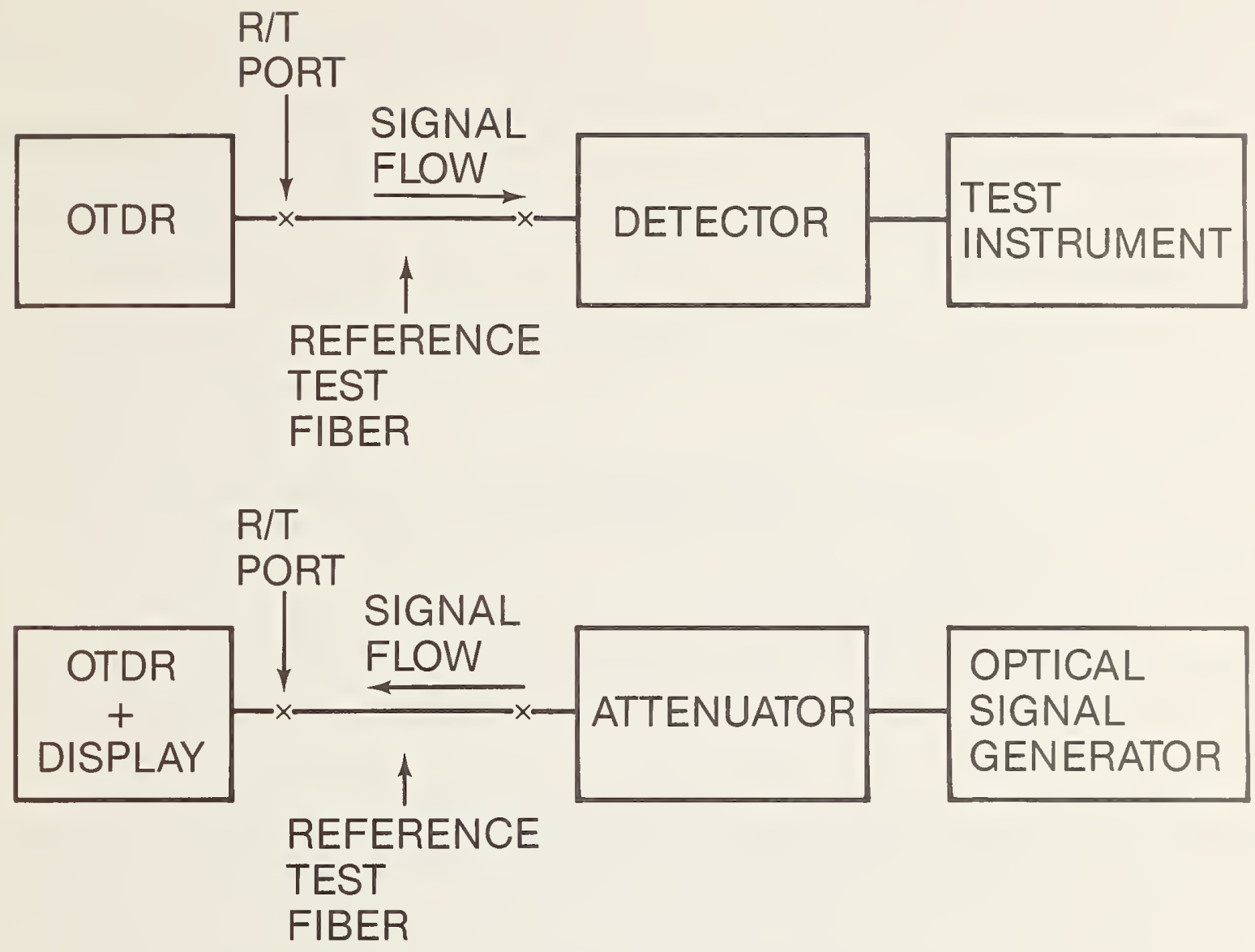

Figure 1 (a). An example of an experimental setup for the active measurement of OTDR characteristics. In order to measure probe peak power, energy, pulse duration, and repetition rate, the test instrument consists of a detector feeding into the appropriate measurement device (oscilloscope, digital counter).

Figure I (b). In (b), the receiver and signal processing characteristics are measured with an optional signal generator and optical attenuator. The optical loss and range scale can be calibrated in this way. The optical source must be synchronized with the OTDR for proper signal processing and display. 
NBS-114A REV. $2 \cdot 8 \mathrm{C}$

U.S. DEPT. OF COMM.
BIBLIOGRAPHIC DATA SHEET (See instructions)

4. TITLE AND SUBTITLE

\section{Calibration and Standardization Issues for the Optical Time-Domain Reflectometer}

5. $A \cup T H O R(S)$

B.L. DanieTson

6. PERFORMING ORGANIZATION (If joint or other than NBS, see instructions)

NATIONAL BUREAU OF STANDARDS

DEPARTMENT OF COMMERCE

WASHINGTON, D.C. 20234

PUBLICATION OR

NBSIR $87-3078$

SPONSORING ORGANIZATION NAME AND COMPLETE ADDRESS (Street, City, Stote, ZIP)

Department of the Navy

Naval Weapons Station/Seal Beach

Corona, California 91720-5000

10. SUPPLEMENTARY NOTES

Document describes a computer program; SF-185, FIPS Software Summary, is attached.

11. ABSTRACT (A 200-word or less factual summary of most significant information. If document includes a significant bibliography or literature survey. mention it here)

We review some of the issues related to the specification and assurance of optical time-domain reflectometer (OTDR) performance. These include selection of appropriate performance parameters, definition of terms, test procedures, measurement difficulties, and use of standard reference fibers. Some recommendations are given for an OTDR calibration program.

12. KEY WORDS (Six to twelve entries; alphabetical order; capitalize only proper names; and separate key words by semicolons)

calibration procedures; optical time-domain reflectometer; OTDR; performance calibrations; standard reference fiber; standard test fiber; text procedures

13. AVAILABILITY

X Unlimited

For Official Distribution. Do Not Release to NTIS

$\square$ Order From Superintendent of Documents, U.S. Government Printing Office, Washington, D.C. 20402.

X Order From National Technical Information Service (NTIS), Springfield, VA. 22161
14. NO. OF

PRINTED PAGES

16

15. Price 



